\title{
Life, the Universe and almost everything
}

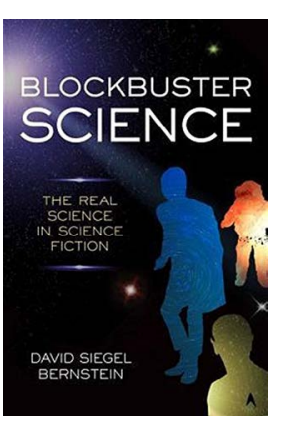

\author{
Blockbuster Science: \\ The Real Science in \\ Science Fiction \\ by David Siegel \\ Bernstein \\ PROMETHEUS BOOKS \\ 2017. 304 PP. $€ 21$
}

T wo decades ago, a 14-year-old Star Trek fan went to the library because she wanted to know what in the Universe a quantum torpedo was. Half a world away and a few years earlier, a 10-year-old obsessed with Jurassic Park just had to know how to build his own dinosaurs. Had David Siegel Bernstein's Blockbuster Science been on the shelves, it would have been one of the books they might have borrowed. It may not have taught her how a quantum torpedo works, nor him how to bring back a T-Rex, but it would have equipped them both with the basics of quantum mechanics and genetics, setting the stage for lives as scientists - and occasional book reviewers.

The breadth of Blockbuster Science is both its strength and its weakness: it gives the reader a good idea of the science behind the science fiction, but at times only scratches the surface. The book covers everything, with a truly encyclopaedic ability to fire example after example from science fiction to illustrate the science being discussed. As the book encompasses the whole spectrum from string theory to genetics to sustainability to AI, Bernstein cannot go into the detailed depths of each topic, so for us (more or less) grown-up scientists the book did sometimes feel a bit shallow - particularly if you know a topic well. On the other hand, we were pleasantly surprised to see very recent topics such as the experimental validation of gravitational waves and the latest research into the near indestructible tardigrade included in the book.

Although there are a lot of references to Star Trek, Star Wars and Doctor Who clearly one of the author's favourites - many less well known TV shows, movies and novels also get a mention. Have a friend who is always on the hunt for more science fiction? Blockbuster Science will give them more to read and watch than they could hope for. Have a friend who is an aspiring science fiction writer? They will love this book as a library of laws, effects and technologies from which they can pick and choose to build their fictional universe.

Because this book is all about the science of science fiction, there are examples that blur the line between the two and really capture the imagination. Indeed, there are current projects developing nanosatellites for an unmanned voyage to our Sun's nearest neighbour. Bernstein, having discussed electronics, robotics and space flight, gives us the Breakthrough Starshot project: the idea of sending spacecrafts with small solar sails - little more than smartphones to Alpha Centauri. Fiction, yes, but not beyond our current technology.

Our favourite nugget of information turned out to be neither physics nor biology, and more closely related to social sciences: civilization rankings.

First, there is the Kardashev scale, which ranks civilizations by their ability to use energy sources, gauging how technologically advanced a civilization is. The scale ranges from type I civilizations, which are able to harness all the power available on a single planet, to type VII civilizations, which are capable of creating universes; basically they are deities. Humans of the twenty-first century are merely a type 0 (or a type 0.7 , if you will, as we can use about $70 \%$ of the Earth's energy resources), while the Gallifreyans - who can harness all the power from the observable universe - make it to type V. The Kardashev scale is fairly wellknown among science fiction readers, and it makes sense to rank technological advance, which usually comes with high demands on energy, by their use of resources, but it's not the only ranking out there.
Bernstein also introduced us to the Barrow scale based on the ability to manipulate their environment and themselves. Here, the lowest rung of the ladder is a type I-minus civilization, which can manipulate objects larger than themselves by building structures or logging wood. This goes up to type

VI-minus civilizations, which can manipulate most elementary particles and organize them into complex systems; but the scale actually extends to type omega-minus, where manipulation of the basic structure of time and space is possible. On the Barrow scale, today's humans are doing pretty well, with our civilization currently transitioning between type IIIminus and IVminus, as we are able to manipulate molecules and in the process of learning to assemble nanotechnology on the atomic scale. We still don't overtake our friends from Gallifrey, though, as they are probably a type omega-minus. Blockbuster

Science doesn't go into enough depth for someone familiar with the science being covered. And there are a few inaccuracies - or perhaps oversimplifications - within its pages, which might confuse readers really unfamiliar with some of the topics. One of us is still not entirely sure what a brane is and the other doesn't quite understand transhumanism. However, if you are looking for a primer on a wide range of sciences and a wide range of science fiction, then Blockbuster Science is your hitchhiker's guide.

\section{NINA MEINZER AND ROSS CLONEY}

Nina Meinzer is an Associate Editor at Nature Communications and Ross Cloney is a Senior Editor at Nature Communications. 